\title{
The Influence of Soviet Oil Painting on Chinese Oil Painting
}

\author{
Li Guang \\ Art Teaching and Research Room, Xijing University, Xian, Shaanxi, China, 710123
}

Keywords: Soviet oil painting; the influence; Chinese oil painting

\begin{abstract}
In the diversity of oil painting, there is positive and important significance in how to rationally treat the influence of Soviet oil painting on Chinese oil painting. This paper, based on the influence of Soviet oil painting on Chinese oil painting, expounds the important influence and function of typical painter's painting on Chinese oil painting, and mainly discusses how to see Chinese oil painting correctly. Only in this way can we treat history correctly. While mastering the art of oil painting, it is necessary to consciously create contemporary Chinese oil paintings with their own national features.
\end{abstract}

\section{Introduction}

The oil painter Perceive the world through theirself works. And at that time, the changes of emotion were transformed into pigments and lines to convey an artistic language. This kind of language can reveal the author's inner world. Based on the influence of Soviet oil painting on Chinese oil painting, we are expounds the important influence and function of typical painter's painting on Chinese oil painting. Based on such background conditions, it is of great significance to study the influence of Soviet oil painting on Chinese oil painting education. Painting is not only the interpretation of the soul, but also the promotion of a kind of culture and connotation. Only by mastering the value of language expression in artistic aesthetics, can we lay a good foundation for the Chinese oil painting education development. So, the next stage of development and practice is the deeper inheritance and development on Soviet oil painting. Which will let Chinese realistic oil painting develop more mature.

\section{The Influence of Soviet Oil Painting on Chinese Oil Painting}

\subsection{The content embodies the true form.}

Oil painting is a reduction of reality. From the selection of oil painting, it comes from life. The content is the nature of all things, which contains the basic emotions of painting. This is one of the important content of the Soviet oil painting. The various forms of language in oil painting show a kind of aesthetic feeling not only limited to visual impact, but also can affect the soul of human soul. In the oil painting language, there are two characteristics. One is freedom and the other is directness. This is not only the outstanding feature of the painting, but also the most basic principle to be followed. For the painter, nature is both the source of creation and the target of creation, which should be based on both nature and nature. Oil painting language is the bridge between the painter and nature. Chinese oil painting focuses on the use of paintings to reflect the painter's view of society and life, which is a free from. Which was also influenced by Soviet oil paintings. Shch as the figure 1 and figure 2 .

\subsection{Colour reflects memory.}

Every painter is an independent individual, and the use of color has his own style. But, the goal is to convey the unique cognition for the viewer. Color is the reaction of the inner world, the painter uses the rational color to show the spirit of his soul. In the oil painting language, the most expressive is the color, which can express the people's thoughts and feelings strongly. The appreciator explores the artist's inner world through the colors of oil painting, understands the emotions and feelings of the painter, who want to become a bosom friend through time and space. 
Under the influence of the Soviet oil painting, Chinese oil painting got better development. At the same time, Chinese painting added his own national element, which according to the main characteristics of Soviet oil painting. This is the difference between Chinese oil painting and other countries, and it is also the real visual presentation of a scene in the deep memory. Shch as the figure 3 and figure 4.

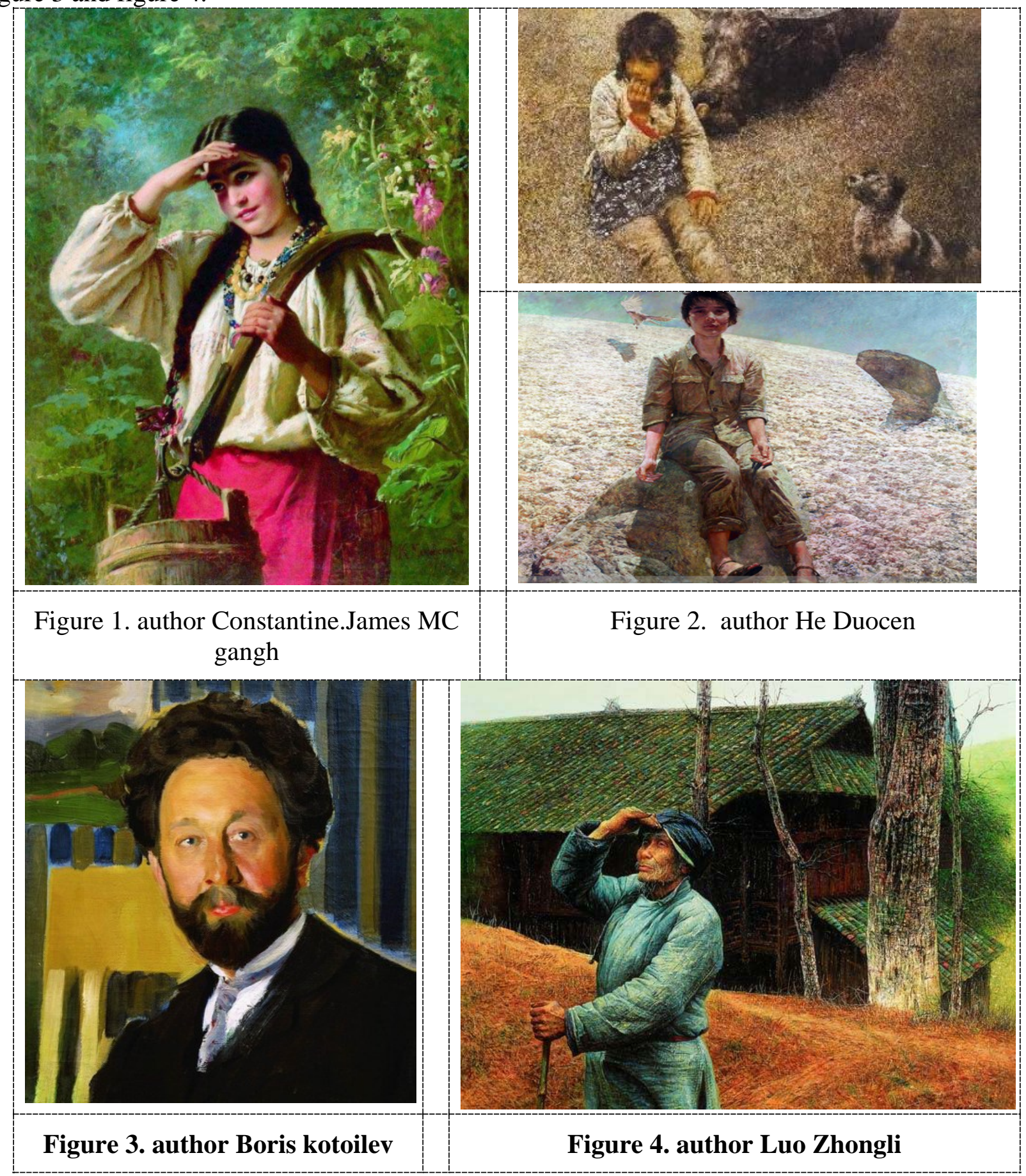

\subsection{Brush strokes show emotion.}

The line is regarded as an important expression technique in painting, and it is also a form of painting language. Because the line is also the display of the creator's heart, and the change of the creator's inner emotions can be achieved by choosing different lines. And how to choose the line will be limit by the painter's creation purpose and method. But the end result is a genuine outpouring of emotion. Shch as the figure 5 and figure 6 . 


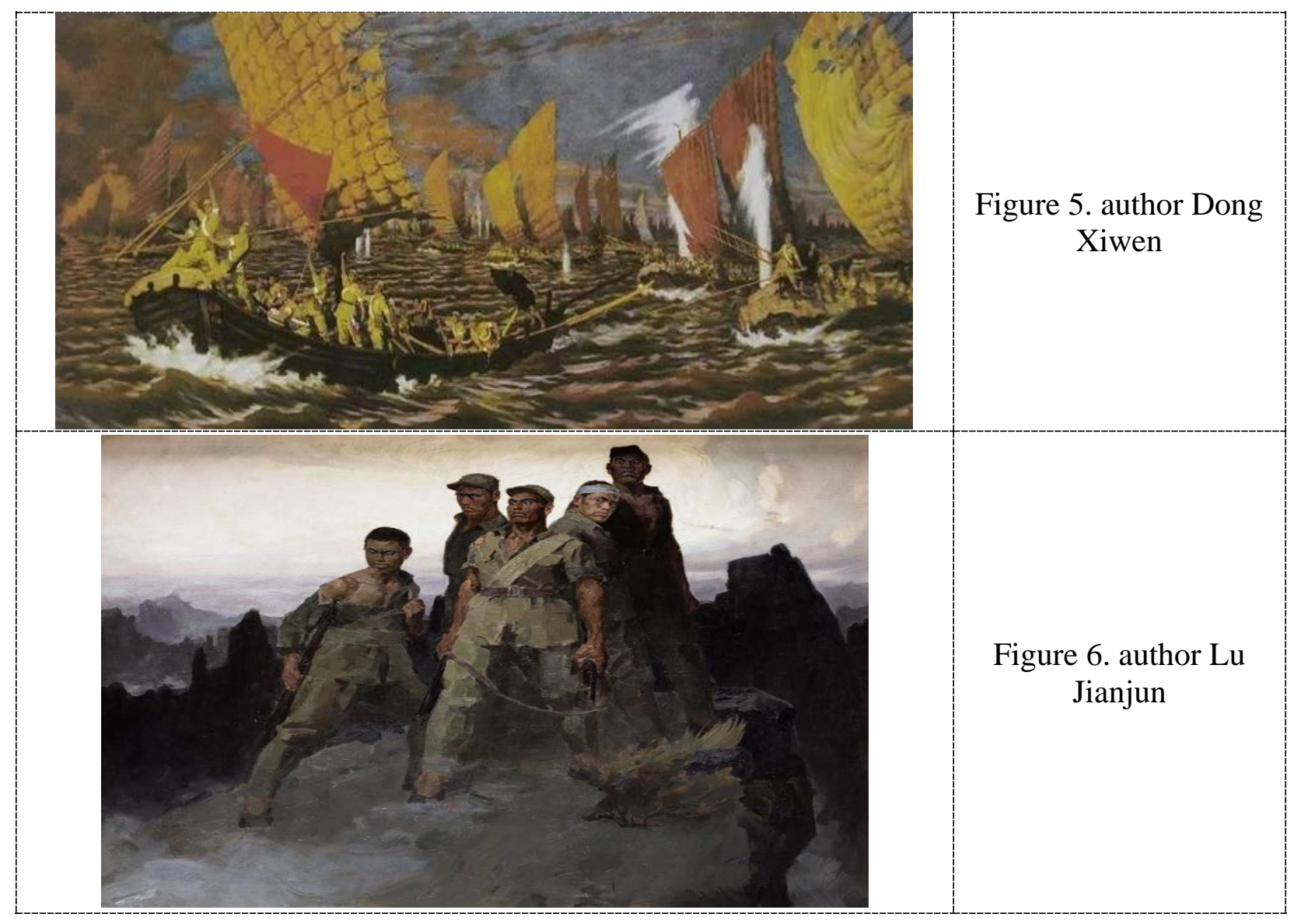

\section{The Rethinking on the Influence}

The influence of Soviet art on Chinese oil painting art in the 1950s and 1960s was comprehensive and in-depth. The creation method of socialist realism has been running through the art creation in this period. The education mode of Soviet art is great significance for the Chinese painters' capacity improving, the enhancement of painting skills and the development of Chinese oil painting art. It has enhanced the painting skills of Chinese painters, and has a far-reaching development for Chinese oil painting art.

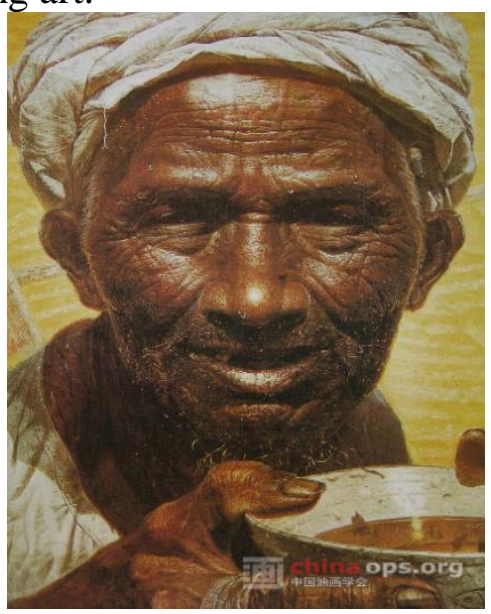

Figure 7. author Luo Zhongli

In the selection of oil painting creation, Soviet socialist realistic painting has a great influence on China. There are the Soviet oil painting character, shch as affirming the reality, praising the positive image of life, expressing the optimism of the people and the vigorous state of spirit, etc. Those aesthetic qualities are yearn for masses of Chinese working people and in harmony with reflecting the reality of life. At the same time, it also accords with the aesthetic requirements of 
"democratization" in Chinese art. So Chinese oil painting creation gradually reflects the real life from the 1950s, such as Luo Zhongli's "father" (figure 7) has revealed a kind of real life breath.

The value of people in Soviet painting must be the place where Chinese oil painting should be studied. The style of Soviet painters is worth studying, shch as Gerasimov, pratov, melnikov, moyshyenko, tkachev brothers and so on. The works have outstanding performance in shaping the vivid images of the characters. From heroic figures to ordinary laborers, from individual characters to major historical themes, all of them are vividly depicted. The great achievements of Soviet painters in oil painting is worth Chinese continuous learning.

\section{Conclusion}

In today's society, the cultural and art fields are developing rapidly. The pattern of cultural and artistic pluralism is formed. The strong modernism trend prevailed, and which lead to some fine arts colleges and universities give up the realistic education system and cultivatecreative and personalized development. This phenomenon has caused great changes in the world art pattern. In the prevailing trend of contemporary art in the world. The study, reservation and development of

the Soviet realistic teaching system will play an important role in maintaining the balance of world art, cultural field, artistic creation and education.

\section{References}

[1] Yulia Karpova 2013 Accommodating 'design': introducing the Western concept into Soviet art theory in the 1950s-60s (Review of History: Revue europeenne d'histoire) pp 214-218

[2] John Fizer 1961 Soviet Art and Soviet Life (The Review of Politics) pp 23-28

[3] Jin Shanshi 2006 Russian painting series (Shandong fine arts publishing house)

[4] Li Youchang 2005 Russia: Russian realistic modeling art museum collection works (Sichuan fine arts publishing house) 